\title{
Healthcare delivery in India amid the Covid-19 pandemic: Challenges and opportunities
}

\author{
PRAGATI B HEBBAR, ANGEL SUDHA, VIVEK DSOUZA, LATHADEVI CHILGOD, ADHIP AMIN
}

\begin{abstract}
In India, the Covid-19 pandemic has thrown open challenges on multiple fronts: (a) the reconfiguration of care in hospitals, in response to Covid-19, has led to many patients suffering nonCovid conditions having to delay their treatment, and (b) the lockdown which though necessary has affected people unequally, some being much worse-off than others. This article unpacks the impact of Covid-19 on healthcare systems in India by raising moral and ethical questions about the plight of patients with other medical conditions while accessing care. This article also proposes a set of actions by which healthcare systems can address Covid and non-Covid related healthcare needs.
\end{abstract}

Keywords: Covid-19, Indian health system, OPD services, teleconsultation, lockdowns, ethical challenges, healthcare access, co-morbidities

\section{Introduction}

Healthcare in the initial days of Covid-19 in India seemed to have taken unpredictable paths for several individuals. A neighbour in Maharashtra had fractured his left thigh bone, a relative in Karnataka had a fall and needed to see an orthopaedician, a chronic diabetic and hypertensive cancer survivor was worried about continuing supply and access to his medications. Both trauma cases with comorbidities had to visit multiple places while in pain, to ultimately be sent home as health facilities were gearing up to cater to Covid-19; while the elderly cancer survivor was able to stock up his medications with the help of his son. The thought of writing

Authors: Pragati B Hebbar (pragati@iphindia.org), PhD scholar and DBT/ Wellcome Trust India Alliance Early Career Fellow, Institute of Public Health, Krishna Rajendra Rd, Banashankari Stage II, Bengaluru, Karnataka 560070 INDIA; Angel Sudha (angelsudha@iphindia.org), Research Officer, Institute of Public Health, Krishna Rajendra Rd, Banashankari Stage II, Bengaluru Karnataka 560070 INDIA; Vivek Dsouza (vivek@iphindia.org),Research Officer, Institute of Public Health, Krishna Rajendra Rd, Banashankari Stage II, Bengaluru, Karnataka 560070 INDIA; Lathadevi Chilgod (latha@iphindia. org), Research Consultant, Institute of Public Health, Krishna Rajendra Rd, Banashankari Stage II, Bengaluru, Karnataka 560070 INDIA; Adhip Amin (adhip@iphindia.org), Research Officer, Institute of Public Health, Krishna Rajendra Rd, Banashankari Stage II, Bengaluru, Karnataka 560070 INDIA

To cite: Hebbar PB, Sudha A, Dsouza V, Chilgod L, Amin A. Healthcare delivery in India amid the Covid-19 pandemic: Challenges and opportunities. Indian J Med Ethics. 2020 Jul-Sep; 5(3) NS: 215-8. DOI:10.20529/IJME.2020.064.

Published online on May 31, 2020.

Manuscript Editor: Olinda Timms

(c) Indian Journal of Medical Ethics 2020 a piece arose as some of us discussed such hardships being faced by people we knew in accessing healthcare, and the fear of falling ill.

Pandemics are not new to humankind, and invariably our health system takes most of the brunt as it caters to existing workload and attempts to cope with the new demand. In the wake of Covid-19, the global community is grappling with attempts to contain the virus as new developments are unfolding rapidly. As per estimates from the 2009 influenza pandemic: school closures, quarantine and social distancing are the most expensive interventions in terms of costs per death prevented, whereas surveillance, contact tracing and face masks were the least expensive (1). India attempted to operationalise several of these measures, but Covid-19 seems to have overtaken the functioning of health facilities while the lockdowns have created additional challenges to seeking routine care.

The question we are trying to understand is 'What is the effect of Covid-19 on the access to healthcare systems in India? (for emergency care, conditions requiring contact with health facilities and follow ups). Searches were conducted in the first week of April 2020, using a variety of sources such as academic literature, media reports, social media, and websites. These searches pointed us towards several difficulties that people are facing in accessing healthcare.

\section{Response of the government and major healthcare entities in tackling non-Covid healthcare needs}

The government from the national to sub-district level swung into action activating the existing systems, developing new systems, absorbing the rapidly changing international guidelines, tapping existing networks and resources. Figure 1 depicts a summary of the detailed guidelines for delivery of essential health services during Covid-19, provided by the Ministry of Health and Family Welfare (MoHFW) on April 14, 2020 (2)

The first case in India was detected on January 30, and lockdown attempted from March 22, yet this guidance was published only on April 14.

Websites of major government and private medical, dental, maternity, cancer and general hospitals were searched for guidelines related to healthcare service delivery. - including the All India Institute of Medical Sciences (Delhi), Christian Medical College and Hospital (Vellore), HealthCare Global, Nephroplus, Clove Dental clinic chain, Jawaharlal Institute of Postgraduate Medical Education and Research (Puducherry), Narayana Health (Bengaluru), Apollo Hospitals, Cloud Nine 


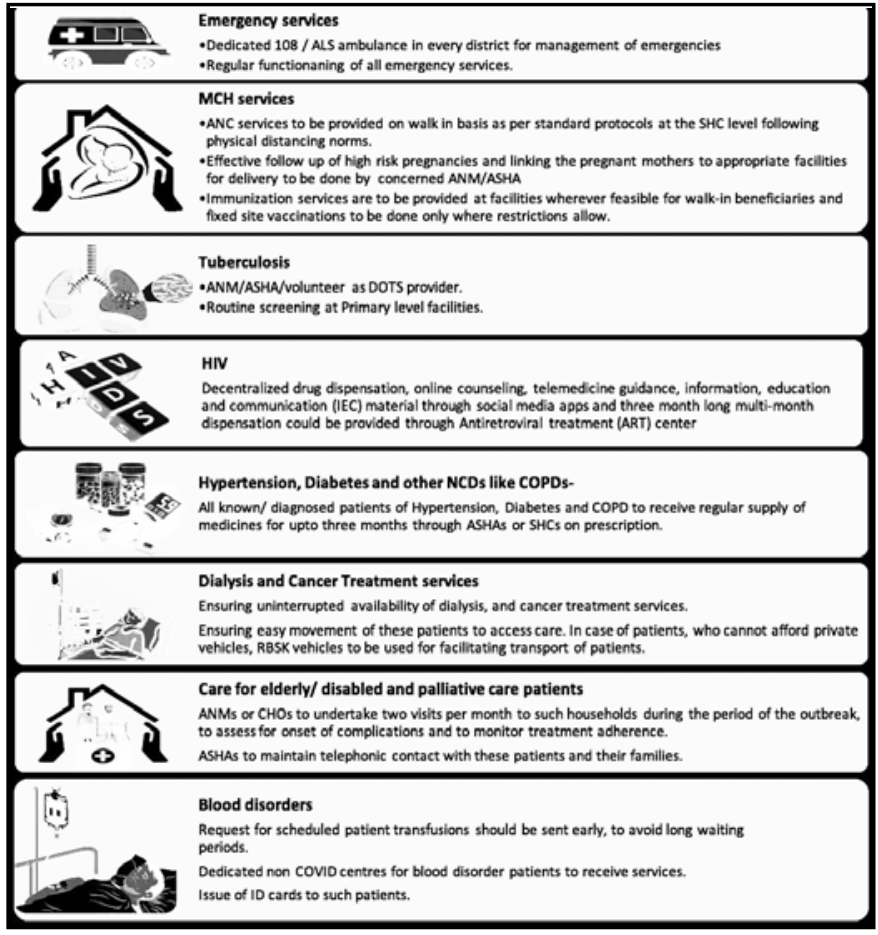

Fig 1: Guidelines for essential health service delivery during Covid-19

Hospital, Tata Memorial Hospital (Mumbai), and associations like the Indian Medical Association, Indian Dental Association, and the MoHFW. A summary of the guidelines issued for essential health services by these major healthcare organisations:

1. Minimising visits to the health facilities as they are encouraging the use of technology via tele/E-consultation, team virtual consultations for OPD services.

2. Emergency services to function normally.

3. Minimal visits to be made by a pregnant mother (based on the mother's condition and as per doctor's advice) were specified by some organisations.

4. Conditions requiring patients to come to the facility, such as radiation therapy, chemotherapy, and dialysis to be continued with necessary precautions to prevent Covid-19 transmission.

Ever since India detected its first Covid-19 case, the country started limiting air travel and issued advisories for health screenings. As the number of cases increased, the government announced its first lockdown on March 22. Since then, India has experienced four such lockdowns - Phase1 (March 24 - Apr 14), Phase 2 (Apr 15 - May 3), Phase 3 (May 4 - May 17), and Phase 4 (May 18 - ongoing). During this period the centre, along with the Health and other ministries, maintained law and order by issuing advisories, notifications, and guidelines for physical distancing, and booking violators for not following protocols. State governments, however, have been largely responsible for arranging and providing service delivery during Covid-19 in close coordination with the centre. In major Indian cities, governments have been working actively in arranging adequate medical staff, hiring healthcare workers (HCWs) and ambulances, opening up new testing centres, procuring PPEs and testing kits, and converting existing public health infrastructure into designated Covid facilities.

\section{Challenges related to healthcare delivery amid Covid-19}

Comorbidities are known to increase vulnerabilities and chances of fatality. Therefore, patients who do not need urgent care, but still require repeated contact with hospitals are in a double bind now. While not getting checked can exacerbate their ill health, hospital visits in Covid-19 times could increase their chances of contracting the virus.

\section{Chronic conditions}

As the pandemic intensifies, many hospitals are advising patients to defer their treatment. According to a media report on April 8, a large cancer specialty hospital in Bengaluru had advised patients undergoing chemotherapy to postpone their treatment by a couple of weeks unless they faced severe symptoms, so as to avoid infection (3). Patients with chronic conditions like HIV, tuberculosis and those requiring dialysis have been struggling to access treatment as hospitals began scaling down their OPDs in response to Covid-19 (4). Previous experiences from the Ebola outbreak suggest that such pandemics can have an impact on both healthcare provision and uptake, especially in areas such as HIV, vaccination, ante and postnatal care, surgeries and consultations, to list a few (5). This is another major challenge for most chronic conditions, including mental health conditions, in detection of new cases and access to medication.

Weighing the risks of delaying treatment versus chances of contracting the infection with compromised immunity is a difficult choice to make; and with restricted access to care providers, patients might have to make such decisions themselves. This touches upon the ethical question of assigning priority such as (i) how to distribute their scarce medical resources among Covid-19 patients; (ii) how to distribute limited resources between Covid and non-Covid patients; and (iii) whether the lockdown as a measure aimed to reduce and prevent the transmissions of Covid-19 infringes the individual right to seek healthcare

Ethically speaking, by leaving patients to decide on the urgency of care that they need, the healthcare system seems to have transferred the burden of ethical and technical decision making onto individuals. Furthermore, in implementing the lockdown asking migrant workers to bear with it, the state has chosen the utilitarian imperative. This is not to criticise the theory of lockdown, which was almost a necessity to contain and slow down the transmission; but rather to question its implementation. Implementation science is imbued with ethical and moral considerations, with distributive justice at its heart, which needs careful deliberation and detailing as can be seen in this case of lockdowns.

In addition to the above dilemmas, there is the issue of allocation of scarce equipment. In Italy for example, the 
guidelines suggest that "In addition to age, doctors and nurses are also advised to take a patient's overall state of health into account...[as]... patients in a worse state of overall health could require a greater share of scarce resources to survive". This is a deeply distressing, ethical call that individuals who are already overworked, and emotionally stretched must make (6). In placing individuals whether healthcare workers, or patients, in such a difficult position, the health system seems to have given up its responsibility.

\section{Acute conditions}

Medical emergencies require immediate medical attention, delay can be life-threatening. The print and social media have highlighted the plight of several patients and their kin where they had to navigate a tortuous path visiting several facilities. Denial of care and deaths due to cancer and cardiac arrests have been reported and also gained attention on social media as among the fallouts of the stringent lockdowns (7). Like the issue of medication for chronic conditions, the issue of availability of blood in blood banks for acute/ emergency conditions is an important requirement and can be a challenge with restricted mobility of blood donors.

Issues faced while seeking care for acute or chronic conditions include the closure of public transport, restriction of use of private vehicles, non-availability of sufficient care providers at the facility and access to admission if required, food and water for patient and caretaker, having necessary prescriptions/ appointment confirmation/ pass to display to the police when traveling to and from the health facility. Therefore, it is not just Covid-19 that has re-oriented care for potentially infected patients, the lockdown has also changed the dynamics of the healthcare system.

\section{Privacy, confidentiality, and stigma}

Artificial intelligence (AI) and app-based technologies are increasingly used to track hotspots, monitor, and understand the nature of Covid-19. However, not having a robust data protection system could do more harm than good. Breaches in confidentiality of patients' health data lead to invasion of privacy, and can exacerbate the degree of stigma within and across communities.

Healthcare workers, and those from vulnerable communities, are discriminated against. Individuals are made scapegoats for the collective fear and anxiety regarding the spread of the infection. There have been several cases where healthcare workers have been harassed by their landlords or neighbours for working in the hospital setting (8). Furthermore, there are reported instances of non-Covid patients who visited a hospital for treatment having been evicted by their house owners. The government has noticed this bigger challenge of fear, mistrust and stigmatisation and has begun promoting positive messaging and gratitude towards health and sanitation workers through updated caller tunes and social media.
Perhaps, it is time that we begin to reflect on two critical aspects - preserving privacy while addressing Covid-19, and looking at social stigma as a public health problem especially in the current context as visiting the healthcare institutions is itself leading to social ostracism.

\section{Maintaining essential services while coping with Covid-19}

Some steps by which health systems can ensure that essential services are not disturbed while dealing with the pandemic:

\section{- Using technology for consultations}

During a pandemic, teleconsultation becomes a lifesaving tool, as it minimises exposure risks for both patients and healthcare professionals (9). Both international and local guidelines are available to implement teleconsultation and telemedicine. For emergency services, it will help in "forward triage" - the sorting of patients before they arrive in the emergency department.

\section{- Improving preparedness of healthcare facilities}

Restructuring of facilities to be able to cater to Covid and non-Covid health needs is crucial to help maintain essential healthcare while minimising infection risks. This could be achieved through physical/ infrastructural changes in the OPD and emergency care set-ups but also through contextualised implementable checklists $(10,11)$.

\section{- Providing medicines at the doorstep}

Online start-ups have been delivering medicines at the doorstep on uploading prescriptions. MoHFW has issued a timely notification on March 26, 2020, specifying guidelines for such deliveries, including prescription validity of 30 days, geographical limits for sellers and mandatory maintenance of bills and details of all transactions (12). Although this may help many, the Internet and Mobile Association of India (IAMAI) has expressed concern that it might restrict delivery of essential medicines during the lockdown (13). The government should streamline this important component of healthcare to ensure adequate access and supply of medications to patients.

\section{- Streamlining travel during the lockdown periods:}

Governments should collaborate with cab and auto services to operate solely for emergency services, thereby mitigating difficulty in accessing care. The government has issued regulations in May 2020, with respect to requirements that the vehicles must have for safe travel such as the number of travellers, provision of hand sanitisers with the driver and the customer, compulsory use of masks, etc. Ola cabs for example in Haryana, and Bengaluru have initiated such an emergency service.

\section{- Effective and transparent communication}

Misinformation in times of a pandemic can lead to fear and undesirable outcomes. Ensuring communication at various levels - within healthcare facilities, from the state 
and central governments to staff on the ground, regular meetings via video conferencing to monitor the situation, with the community to inform and create awareness - is crucial. Checking on the mental and physical health of staff should be made a routine. Optimising existing systems, such as short announcements by the waste collection trucks of key messages in the local language, is another low-cost method to reach every household.

This article attempts to provide insights into how Covid-19 has impacted the Indian health system, what measures have been taken proactively, and what could be potential solutions and focus areas for the coming days. As the health system adapts to cope with this pandemic, it needs to be mindful of the several moral and ethical dilemmas that may occur during patient care, confront stigma-related issues that would arise for patients as well as care providers, provide for the vulnerable groups who may not be able to access healthcare but require it the most, and conceptualise a post-Covid era to be able to survive the storm and still sustain itself.

Statement of competing interests and funding support: The authors have no competing interests to declare. No funding was received for this article, but the authors' time was supported by their respective projects as follows: Pragati Hebbar and Vivek Dsouza through the DBT/Wellcome Trust India Alliance Fellowship (IA/ CPHE/17/1/503338) awarded to Pragati. Adhip Amin through the DBT/Wellcome Trust India Alliance Fellowship (IA/CPHI/17/1/503346) awarded to Upendra Bhojani. Angel Sudha through the Institute of Public Health (IPH) for learning and professional development. Lathadevi Chilgod through the National Health Systems Resource Centre (NHSRC) - Innovations \& Learning Centre.

Acknowledgements: The authors would like to acknowledge the contribution of Mr Praveen Rao who helped design Figure 1, and comments on a previous version of this article by Dr. Prashanth NS as part of peer review within IPH.

\section{References}

1. Madhav N, Oppenheim B, Gallivan M, Mulembakani P, Rubin E, Wolfe N. Pandemics: Risks, impacts, and mitigation. In: Jamison DT, Gelband $\mathrm{H}$, Horton S, et al., editors. Disease Control Priorities: Improving Health and Reducing Poverty. 3rd edition. Washington (DC): The International Bank for Reconstruction and Development / The World Bank; 2017 Nov 27[cited 2020 May 14]. Chapter 17. Available from: https://www.ncbi.
nlm.nih.gov/books/NBK525302/

2. Ministry of Health and Family Welfare, Govt of India. Enabling Delivery of Essential Health Services during the COVID 19 Outbreak Guidance note. New Delhi: MoHFW; 2020 Apr 14[cited 2020 Apr 16]. Available from; https://www.mohfw.gov.in/pdf/ EssentialservicesduringCOVID19updated0411201.pdf

3. Kulkarni T. Cancer patients worried as hospitals focus on COVID-19. The Hindu 2020 Apr 8[cited 2020 Apr 16]. Available from: https://www. thehindu.com/news/cities/bangalore/cancer-patients-worried-ashospitals-focus-on-covid-19/article31292061.ece/amp/

4. Johari A. India's focus on coronavirus leave TB and HIV patients adrift. Scroll. 2020 April 7 [cited 2020 Apr 16]. Available from: https://scroll.in/ article/958400/invisible-crisis-tb-and-hiv-patients-left-adrift-in-indiasfocus-on-coronavirus

5. Ribacke KJB, Saulnier DD, Eriksson A, Schreeb JV. Effects of the West Africa Ebola virus disease on health-care utilization - A systematic review. 2016 Oct 10[cited 2020 Apr16]. Front Public Health. 4:222. Available from: 10.3389/fpubh.2016.00222

6. Mounk Y. The extraordinary decisions facing Italian doctors. The Atlantic. 2020 Mar 11[cited 2020 Apr 16]. Available from: https:// www.theatlantic.com/ideas/archive/2020/03/who-gets-hospitalbed/607807/

7. Jain P. Heart attack patient denied treatment in Delhi. India Today. 2020 April 8 [cited 2020 Apr 17]. Available from: https://www. indiatoday.in/india/story/heart-attack-patient-denied-treatment-indelhi-1664652-2020-04-08

8. News18. Day after honouring doctors with claps, many in India are evicting them fearing Covid-19. News 18. 2020 Mar 24 [cited 2020 Apr 17]. Available from: https://www.news $18 . c o m / n e w s / b u z z / d a y-$ after-honouring-doctors-with-claps-many-in-india-are-evicting-themfearing-covid-19-2548937.html

9. Hollander JE, Carr BG. Virtually perfect? Telemedicine for Covid-19. New Engl J Med. 2020 Apr; 382:1670-81.

10. COVID-19-PHC Action Group. COVID-19 Preparedness Checklist for Urban Primary Health Centres in in India. 2020 Apr 20 [cited 2020 Apr 17]:v1.0. Available from: https://iphindia.org/wp-content/ uploads/2020/04/COVID-19-Preparedness-Checklist-for-UPHCs-inIndia-v1.0.pdf

11. Covid-19-PHC Action Group. COVID-19 Preparedness Checklist for Rural Primary Health Care and Community Settings. 2020 Apr 3:v1.1 Available from: https://iphindia.org/wp-content/uploads/2020/04/ COVID-19-Preparedness-guidance_checklist-for-rural-Primary-HealthCare Community-ealth-settings-in-India-v1-1.pdf

12. Ministry of Health and Family Welfare, Govt of India. Notification regarding Doorstep delivery of drugs. New Delhi: MoHFW; 2020 Mar 26[cited 2020 Apr 16]. Available from: https://www.mohfw.gov.in/pdf/ Doorstepdelivery26B.pdf

13. Mandavia M. Restrictions on online sale of drugs to hurt e-pharmacies, says IAMAI. Economic Times. 2020 Mar 30[cited 2020 Apr 17]. Available from: https://economictimes.indiatimes.com/industry/ healthcare/biotech/healthcare/iamai-writes-to-health-ministryagainst-new-rules-allowing-doorstep-delivery-of-medicines/ articleshow/74896361.cms?utm_source=contentofinterest\&utm_ medium=text\&utm_campaign=cppst 